\title{
CARCINOMA IN SITU Y EMBARAZO
}

\section{Prof. Rubén Darío Guzmán A.* Prof. Alvaro Fonnegra M.**}

Desde la utilización en nuestro medio de la citología vaginal rutinaria en la consulta prenatal, el diagnóstico del cáncer cervical en la mujer embarazada es hoy en día más frecuente. Antiguamente en nuestro servicio un gran porcentaje de $\mathrm{Ca}$. in situ del cérvix en la mujer gestante pasaba inadvertido, pero hoy gracias a este procedimiento hemos podido estudiar, diagnosticar y tratar en un lapso de 11 años, 13 casos de Ca. preinvasivo, en los servicios de Ginecología del Hospital San Juan de Dios y del Instituto Materno Infantil Concepción Villaveces de Acosta, del 1? de Enero de 1960 al 30 de Septiembre de 1971. La gran mayoría de ellos corresponde a los últimos años, cuando nuestros residentes y nosotros mismos, hemos tratado de pesquisar hasta la saciedad tal afección.

Siempre han existido discusiones importantes en relación con los cambios cervicales, patogenia, diagnóstico y tratamiento del $\mathrm{Ca}$. in situ concomitante con la gestación; sin embargo, los trabajos de Greene y Peckham (1), Hamperl y Kaufman (2), Marcus (3), Marsh y Fritzgerald (4), Moore y Gusberg (5), Chung, Bayly y Benaren (6), Richard (7), han aminorado tal inquietud relacionada sobre los cambios que el influjo hormonal pueda producir sobre el cuello de la mujer embarazada.
La mayoría de los autores están de acuerdo en que el estudio citológico es tan fidedigno en la embarazada como en la no embarazada, es decir, que las atipias más importantes del cuello uterino descubiertas durante la gestación persisten en el post-parto, como lo admiten Slate, Martin y Merrit (8), Ferguson y Cavanagh (9), Ayre (10). Referente al tratamiento también existen discrepancias, como lo pudimos exponer en un trabajo anterior publicado en la Revista de Obstetricia y Ginecología en 1968 (11); sin embargo, la mayoría de los autores son partidarios de tener biopsias, seguidas de conización del cuello uterino en cualquier época del embarazo y permitir el parto por las vías naturales; los casos que no sigan esta norma se estudiarán más tarde a partir de la 4a o 6a semana del puerperio, conducta que seguimos actualmente en el servicio. La frecuencia es muy variable, en general oscila del $2,3 \% 0$ al $2,4 \% 0$.

\section{Material y Métodos}

Se analizan y estudian 13 casos de Ca. in situ del cuello uterino comitantes con embarazo, en un lapso de

\footnotetext{
* Profesor Asistente, Dpto. de Obstetricia y Ginecología, Facultad de Medicina U. N.

** Profesor Asociado, Depto. de Obstetricia y Ginecología, Facultad de Medicina U. N.
} 
11 años, en los servicios de Ginecología del Hospital San Juan de Dios de Bogotá y del Instituto Materno Infantil "Concepción Villaveces de Acosta". En algunos casos la conducta fue errónea por la poca experiencia que teníamos en ese entonces relacionada con el manejo de estas enfermas.

PARIDAD :

Multíparas:
La incidencia total corresponde a
las multíparas, lo cual está de acuer-
do con lo encontrado por todos los
autores.
EDAD :
Entre 26 y 35 años:
Entre 36 y 45 años:

El mayor número de $\mathrm{Ca}$. de cérvix concomitante con embarazo se encontró en las mujeres entre los 26 y los 35 años, lo cual también está de acuerdo con los datos de los demás autores. Ningún caso se encontró en las enfermas por debajo de los 25 años, motivo que nos hace pensar que las campañas con el fin de descubrir el carcinoma de cuello uterino debiera desarrollarse en mujeres mayores de los 25 años.

\section{Motivo de Consulta:}

Algunas mujeres no presentaban síntomas en el momento de acudir a la Consulta Prenatal, otras discreta hemorragia genital, otras flujo genital y algunas, síntomas relacionados a su estado grávido.

\section{ESTADO DEL CUELLO UTERINO:}

Erosionado:

Ectropión :

Aparentemente sano:
Hubo 6 casos con cuello aparentemente sano, en donde la citología exfoliativa desempeñó un papel de gran importancia para descubrir tal afección. Recordar que el $\mathrm{Ca}$. in situ puede presentarse en el cuello de la mujer embarazada en un epitelio cervical sano.

\section{CITOLOGIAS :}

\begin{tabular}{llrr}
$\mathrm{A}-3$ & $=$ & 1 & $8 \%$ \\
$\mathrm{~A}-4$ & $=$ & 7 & $54 \%$ \\
$\mathrm{~A}-5$ & $=$ & 4 & $38 \%$ \\
No se hizo & $=$ & 1 & $8 \%$ \\
& & $\frac{1}{13}$ & \\
\cline { 3 - 4 } & & &
\end{tabular}

Es llamativo que no se presentó ningún caso de falso negativo y la mayoría de las citologías correspondieron a las A4 y A5; esto demuestra la eficacia de este procedimiento en la investigación y pesquisa del $\mathrm{Ca}$. de cérvix.

BIOPSIA DE CUELLO:

\begin{tabular}{llrr} 
Ca. in situ & $=$ & 10 & $77 \%$ \\
No se efectuó & $=$ & 3 & $23 \%$ \\
\cline { 2 - 3 } & & $100 \%$
\end{tabular}

En 10 enfermas se practicó biopsia cervical siendo todas positivas para Ca. in situ. En tres no se efectuó; una llegó en trabajo de parto y en las dos restantes el obstetra no consideró necesario tomarlas, por tratarse de cuellos aparentemente sanos.

EMBARAZO EN SEMANAS:

$\begin{array}{rrrrlrl}\text { De } & 8 & \text { a } & 10 & \text { semanas: } & 3 & 23 \% \\ \text { De } 11 & \text { a } & 20 & \text { semanas: } & 2 & 15.5 \% \\ \text { De } 21 & \text { a } & 30 & \text { semanas: } & 2 & 15.5 \% \\ \text { De } 31 & \text { a } & 40 & \text { semanas: } & 6 & 46 \% \\ & & & & 13 & 100 \%\end{array}$

La mayor incidencia se encontró entre la 31 a la 40 semanas de embarazo. 


\section{CONIZACIONES :}

$\begin{array}{llrr}\text { Antes del parto } & = & 10 & 77 \% \\ \text { En el puerperio } & = & 2 & 15 \% \\ \text { No se efectuó } & = & 1 & 8 \% \\ & & \frac{1}{13} & 100 \%\end{array}$

Dos conizaciones se efectuaron en el puerperio: una porque la enferma ingresó al servicio con un embarazo muy avanzado (39 semanas) y las condiciones del cuello uterino no eran las requeridas para efectuar una conización; la otra porque la enferma presentaba un feto en situación transversa, motivo por el cual se le practicó una cesárea y luego se conizó en el puerperio. La que no se conizó se debió a que llegó en trabajo de parto y posteriormente presentó gran hemorragia abdominal con hipofibrinogenemia motivo por el cual hubo que practicarle una histerectomía abdominal total, encontrándose en Ca. in situ de cérvix.

\section{SEM:ANAS EN QUE REALIZARON LAS CONIZACIONES :}

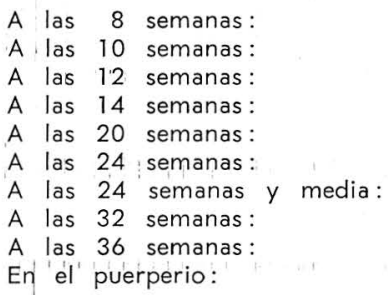

En algunas ocasiones la conización antes del parto puede producir complicaciones como aborto, amenaza de parto prematuro, hemorragias; algunos autores como Rad (12), consideran que vale la pena correrlos y no dejar evolucionar un carcinoma invasivo, en el cual el tratamiento del parto sería de consecuencias funestas. Solamente en un sólo caso una enferma presentó hemorragia abundante a los 2 y 3 meses después de la conización, presentando un aborto en la última hospitalización; otra presentó muerte fetal in útero dos meses después de la conización, motivo por el cual hubo que inducirla para que abortara. Este último autor presenta 38 casos de conización y embarazo, 4 de los cuales terminaron en abortos y 1 presentó una hemorragia tardía diez días después de la conización. Gallegos en Venezuela (13), es partidario de la conización antes de la 34 semanas del embarazo. Presenta 12 casos de conizaciones ( 6 en el primer trimestre y 6 en el segundo), dos de los cuales presentaron hemorragia abundante; en ningún caso hubo interrupción del embarazo, ni complicaciones en el parto.

\section{Tumor Residual}

Solamente hubo un caso de Tumor Residual tanto en el cono como en la Histerectomía abdominal. Esto está de acuerdo con Rutledge y colaboradores (14), quienes en estudio de 3.873 embarazadas no encontraron caso alguno de lesión residual en el post-parto; no obstante Way (15), informa $90 \%$ de carcinoma in situ residual en histerectomías ulteriores al embarazo.

\section{CONDUCTA OBSTETRICA :}

Cesárea más histerectomía abdominal ampliada $=4$

$38 \%$

Cesárea, seguida de Conización al puerperio $=2$

$15 \%$

Cesárea $=1$

$8 \%$

Histerectomía abdominal ampliada $=1$

$8 \%$

Conización, seguida de parto, por las vías naturales $=2$

Conización, seguida de Revisión Uterina por aborto incompleto $=1$

Conización $=2$

$100 \%$

Mortalidad: En ningún caso hubo muerte materna. 
ESTADO FETAL :

\begin{tabular}{lrr} 
Vivos : & 10 casos & $77 \%$ \\
Abortos : & 3 casos & $23 \%$ \\
\cline { 2 - 3 } & -13 & $100 \%$
\end{tabular}

Esto demuestra la poca repercusión que tiene el $\mathrm{Ca}$. in situ sobre la vida del feto.

CONTROLES :

\begin{tabular}{lrr} 
Hasta un año & 7 & $54 \%$ \\
Recientes & 3 & $23 \%$ \\
Sin control & 3 & $23 \%$ \\
\cline { 2 - 3 } & 13 & $100 \%$
\end{tabular}

A dos pacientes de las recientes se les tiene citadas para practicarles histerectomía abdominal en el puerperio.

Sabemos lo difícil que son en nuestro medio los controles debido a la idiosincrasia de nuestras pacientes, motivo por el cual existen 7 pacientes que se intervinieron hace más de un año y no han vuelto y 3 que definitivamente no se supo de ellas una vez que salieron del servicio.

\section{Resumen y Conclusiones}

1. Se analizan y estudian 13 casos de Ca. in situ concomitantes con embarazo en un lapso de 11 años del servicio de Ginecología del Hospital San Juan de Dios y del Instituto Materno Infantil "Concepción Villaveces de Acosta" de Bogotá.

Encontramos 6 pacientes $(40 \%)$ con cuello aparentemente sano; esto nos pone de presente la importancia de la citología exfoliativa en la pesquisa del $\mathrm{Ca}$. de cérvix.

De los 13 casos, en 10 se practicó biopsia de cuello, siendo todas positivas para $\mathrm{Ca}$. in situ de cérvix. En 3 pacientes no se efectuó.
Se practicaron 12 conizaciones: 10 antes del parto (desde la 8a hasta la 36 semanas), y 2 en el puerperio; en una no se practicó.

Solamente hubo un solo caso de tumor residual tanto en el cono como en la histerectomía abdominal ampliada.

Se expone la conducta obstétrica que siguió en todos los casos, siendo la más frecuente la de cesárea más histerectomía abdominal ampliada (38\%).

No se presentó ningún caso de mortalidad materna.

En cuanto al estado fetal, obtuvimos 10 fetos vivos. El aborto estuvo presente en 3 casos.

Los controles fueron relativamente deficientes debido a la idiosincrasia de nuestras pacientes. Tres enfermas no volvieron a control; otras 13 pacientes se encuentran recientemente intervenidas en el servicio.

\section{Summary and Conclusions}

1. Thirteen cases of concomitant Ca. in situ, with pregnancy, are analized and studied during a period of 11 years at the gynecological ward of Hospital San Juan de Dios and of Instituto Materno Infantil "Concepción Villaveces de Acosta" in Bogotá.

We encountered six patients with cervic apparently healthy; the latter serves to note the importance of exfoliative cytology when in search of a cervical ca.

A biopsy of the cervix was performed in 10 of the 13 cases, being the results all positive for ca. in situ of the cervix. This was not performed in three cases.

Twelf conifications were accomplished: 10 prior to birth (from the 
8th. to the 36th. week) and 2 during puerperium; not performed in one case.

There was only one case of residual tumor both in the cone as in the enlarged abdominal hysterectomy.

The obstetrical method folloved in all cases is explained, being cesarotomy more frequent than enlarged abdominal hysterectomy (38\%).

There was no case of maternal mortality.

With respect to the fetal status, we obtained 10 alive. Abortion was present in three cases.

The controls were relatively deficient due to the idiosyncracy of our patients. Three patients did not return for control; another 13 patients have been recently operated on in the ward.

\section{BIBLIOGRAFIA}

1 GREENE, R. R. y cols. y PECKMAN, B. M. : Am. J. Obst. \& Gynec. 75: 551, 1958.

2 HAMPERL, H., y KAUFMAN, C. y OBER, KG. Archiv, Gynak, 181, 184, 1954.

3 MARCUS, M. B., BRAND, M. L. Y CIBLEY, L. J.: Obst. \& Gynec. 10: 669, 1957.
4 MARSH, M. y FRITZGERALD, P. J.: Cancer. 9: 1195, 1956.

5 MOORE, D. B. Y BUSPERG, S. B.: Obst. \& Gynec. 18: 530, 1959.

6 PECKMAN, B., GREENE, R., CHUNG J. R., BAYLY, H. A. y BENAREó, H. B. W.: Am. J. Obst. \& Gynec. 67: 21, 1954.

7 RICHARD R.: Am. J. Obst. \& Gynec. 87, 474, 1963.

8 SLATE, T. A., MARTIN P. L. Y MERRIT J. W.: Am. J. Obst. \& Gynec. 74: 344, 1956.

9 FERGUSON Y CAVANAGH: Am. J. Obst. \& Gynec. 344, 1956.

10 AYRE, J. E. Obst. \& Gynec. 76: 780, 1958.

11 GUZMAN ALANDETE RUBEN DARIO. Carcinoma In situ y Embarazo. Rev. Col. de Obst. y Ginecología. Vol. XIX. No 5 Sept. y Octubre de 1968.

12 RAD: RAD, M. MARCZINKE I., BOYES. D. A. FIDLER, H. K.: El uso de la Citología exfoliativa durante el embarazo. Am. J. Obst. \& Gynec. Memorias de la XXIII de la Soc. de Obst. y Gynec. del Canadá. Vol. 94, № 4 de Febrero de 1966.

13 GALLEGOS y cols. : Ca. in situ. Rev. Obst. y Gin. de Venezuela. 27: 261, 1966.

14 RUTLEDGE, C. E. Jr. Christopherson, W. M. y PARKER, J. E.: Cervical displasia and carcinoma in pregnancy. Obst. \& Gynec. 19: $351,1962$.

15 WAY, S.: Exfoliative cytology, early carcinoma of the cervix, pregnancy and fertility. Acta cytologica, 6: 547, 1962. 